\title{
Documento
}

\section{UNIONES EN HENDIDURA Y SU PAPEL FUNCIONAL EN EL TRACTO REPRODUCTOR FEMENINO}

\author{
Drs. María C. Brañes O.1a, Alejandra Sáez R.2, Manuel J. Villalón B. ${ }^{1 b}$, Juan C. Sáez C.1a \\ ${ }^{1}$ Departamento de Ciencias Fisiológicas, Facultad de Ciencias Biológicas, Pontificia Universidad Católica de Chile. \\ ${ }^{2}$ Médico, Servicio de Obstetricia y Ginecología, Hospital Gustavo Fricke
}

aBioquímico, Ph.D. bLicenciado Ciencias Biológicas, Ph.D.

\section{RESUMEN}

En el aparato reproductor femenino se expresan diferentes conexinas (Cxs), proteínas que forman canales de uniones en hendidura $(\mathrm{CUH})$ entre células en contacto, permitiendo la coordinación de respuestas metabólicas y/o eléctricas de grupos celulares. Los CUH jugarían un papel relevante en el desarrollo de las células de la granulosa, ya que permiten la comunicación heteróloga entre el ovocito y las células del cúmulo manteniendo la detención meiótica. En la trompa de Falopio, los CUH coordinarían el batido ciliar del epitelio y la contracción muscular, facilitando el desplazamiento de los gametos y del embrión. En el útero, los CUH conectan a las células miometriales y también a las endometriales. El aumento de CUH durante el preparto permitiría la contracción uterina coordinada facilitando el trabajo de parto al término del embarazo. La expresión de las Cxs es regulada por hormonas, lo que explicaría el perfil de CUH presentes en los diversos tipos celulares del tracto genital en diferentes estadios fisiológicos del sistema reproductor.

\section{PALABRAS CLAVES: Canales intercelulares, conexinas, útero, oviducto, ovario}

\section{SUMMARY}

Several connexins, protein subunits that constitute gap junction channels (GJC), are expressed in the female reproductive tract allowing coordination of metabolic and/or electrical responses. The GJC could play relevant roles in the development of granulosa cells, since they permit heterologous oocyte-cumulus cell communication maintaining the meiotic arrest. In the Fallopian tube, GJC would coordinate the epithelial ciliary beat and muscle contraction, favoring the transport of gametes and embryos. In the uterus, GJC connect myometrial, as well as, endometrial cells. The increase in intercellular communication via GJC before labor would allow coordinated uterine contraction, favoring labor at term pregnancy. The expression of connexins is hormonally regulated and seems to explain the pattern of GJC expression in different cell types of the reproductive system at different physiological stages.

KEY WORDS: Intercellular channels, connexins, uterus, oviduct, ovary 


\section{INTRODUCCION}

Los canales intercelulares, también llamados canales de las uniones en hendidura $(\mathrm{CUH})$, son permeables a iones y moléculas pequeñas (menores de $1,2 \mathrm{kDa}$ ) y comunican el citoplasma de células adyacentes, permitiendo la coordinación de respuestas (metabólicas y eléctricas) de un conjunto de células frente a un determinado estímulo, tanto fisiológico como fisiopatológico. Cada $\mathrm{CUH}$ lo constituyen dos hemicanales o conexones, cada uno es aportado por una de las dos células en contacto. A su vez, cada hemicanal está compuesto por seis subunidades proteicas denominadas conexinas (Cxs). Las Cxs son codificadas por una familia de genes compuesta por al menos 20 genes diferentes en el humano y se denominan con el prefijo $\mathrm{Cx}$ seguido de un número que indica el peso molecular teórico aproximado (Figura 1).

Cada célula puede expresar más de una $\mathrm{Cx}$, lo que amplía la gama de variantes de conexones y/ o canales intercelulares posibles de formar, cada uno con características de permeabilidad y de regulación propias, que parecen adaptarse a las necesidades funcionales de cada tipo celular que las expresa. Todas las Cxs hasta ahora estudiadas, a excepción de la Cx26, son fosfoproteínas y el estado de fosforilación de algunas de ellas regula diversas propiedades de los $\mathrm{CUH}$ que forman, incluyendo la apertura. Otros mecanismos, tales como transcripción génica, traducción proteica, ensamblaje del conexón, su traslocación hacia la membrana o la internalización del canal desde la membrana constituyen otros mecanismos que regulan el grado de comunicación intercelular. En general, las Cxs presentan una vida media corta (2-3 h), sugiriendo que presentan una alta tasa de recambio o que se pueden expresar transitoriamente (1). La coordinación de respuestas celulares a través de CUH se logra mediante la transferencia de moléculas de señalización intracelular, como nucleótidos cíclicos e inositol trifosfato, y/o el paso de corriente. Por ejemplo, los CUH son esenciales en la propagación intercelular de un tipo de ondas de $\mathrm{Ca} 2+$ que coordinan respuestas metabólicas de hepatocitos en el hígado y permiten la tranferencia intercelular de cambios de potencial de membrana que gatillan la propagación de una onda de contracción en el miocardio.

El papel fisiológico de los $\mathrm{CUH}$ en varios tejidos ha sido también revelado por el descubrimiento de mutaciones en las Cxs asociadas a las enfermedades genéticas humanas, tales como la

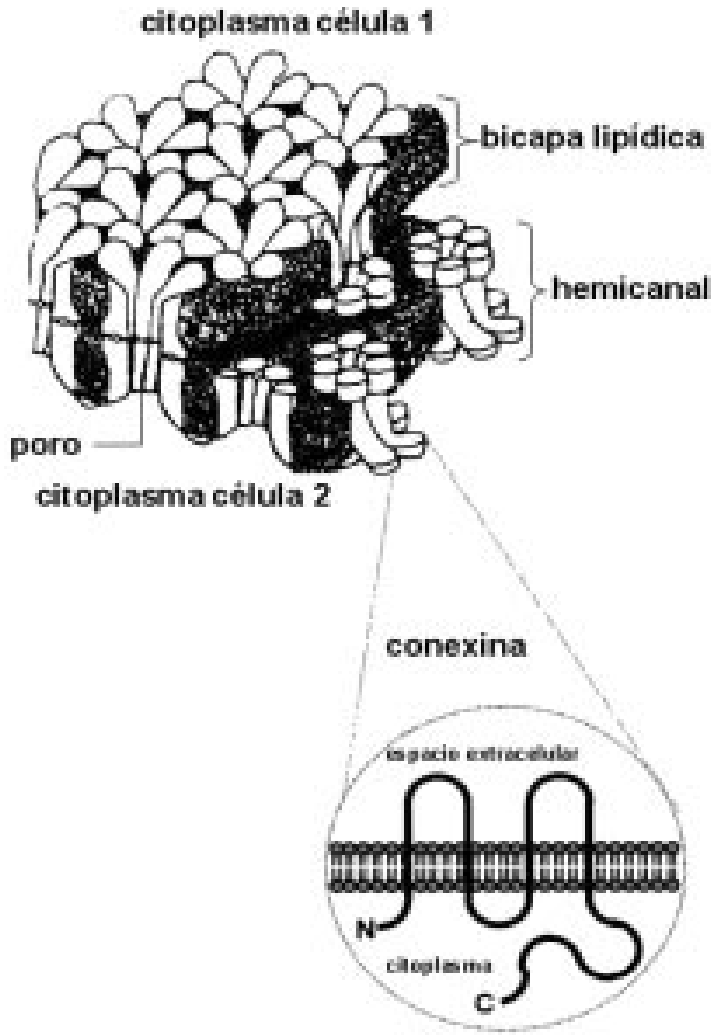

Figura 1. Esquema de una placa de unión en hendidura compuesta por una aglomeración de canales de comunicación intercelular o de uniones en hendidura (UH). El nombre unión en hendidura describe su apariencia vista por microscopía electrónica de cortes finos, sin embargo, estructural y funcionalmente no existe la hendidura o espacio entre los dos hemicanales. El esquema muestra que cada hemicanal inserto en la membrana celular está formado por seis subunidades de conexina. Un hemicanal en la célula 1 encuentra otro hemicanal en la célula 2 adyacente, dando origen a un canal intercelular que comunica los citoplasmas de las dos células en contacto. Se incluye la organización topológica de una conexina en la bicapa lipídica, con cuatro segmentos transmembrana y con su extremo amino $(\mathrm{N})$ y carboxilo (C) terminal en el lado citoplasmático de la membrana celular.

neuropatía demielinizante conocida como CharcotMarie Tooth ligado al cromosoma $X$, algunas sorderas, enfermedades de la piel y cataratas (1). Además, la generación de ratones transgénicos con deleción o inactivación de genes de Cxs muestran fenotipos que van desde la disfunción tejido específico hasta la mortalidad perinatal, dependiendo de la importancia funcional del tipo celular que la expresa (2). 


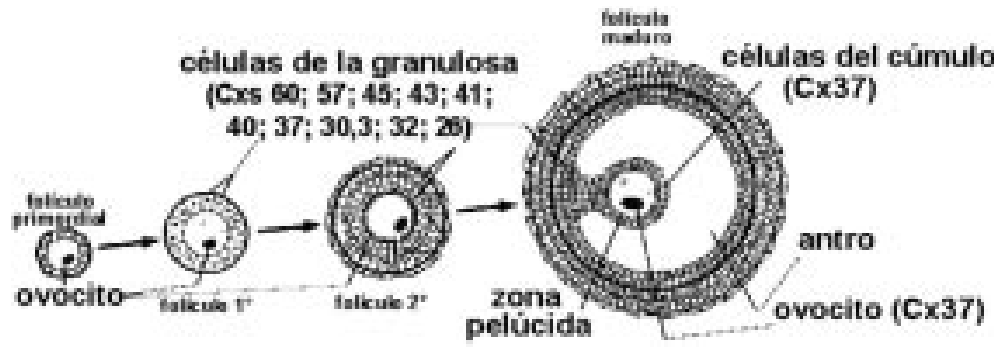

Figura 2. Diagrama que muestra etapas del desarrollo folicular y las conexinas (Cxs), estudiadas hasta la fecha, que se expresan en cada uno de los tipos celulares del folículo ovárico.

\section{CUH Y CXs EN EL FOLÍCULO OVÁRICO}

Estudios ultraestructurales han revelado la presencia de CUH entre las células foliculares de la rata $(3,4)$. Además se han identificado varias Cxs en diferentes especies de mamíferos, incluyendo a la Cx26, Cx32, Cx30.3, Cx37, Cx40, Cx41, Cx43, Cx45, Cx57 y Cx60 (5-14). La Cx43 es muy abundante y parece constituir una de las vías más importante en la conexión entre las células de la granulosa (Figura 2) $(5,15,16)$. La Cx43 en el ovario es regulada durante el desarrollo y por los niveles de hormonas sexuales. En efecto, la Cx43 se expresa tanto en etapas tempranas del desarrollo embrionario (17), como durante la madurez sexual (9). Durante el crecimiento folicular se produce un ciclo de expresión de la $\mathrm{Cx} 43$, en donde la cantidad de Cx43 aumenta previo a la ovulación $\mathrm{y}$, disminuye al aumentar los niveles de $\mathrm{LH}$ y durante la atresia folicular $(16,18,19,20)$. Además, se ha detectado CUH y $\mathrm{Cx} 43$ en el cuerpo lúteo (Figura 3) $(21,22)$, donde podrían jugar un papel relevante en el crecimiento, diferenciación y regresión del cuerpo lúteo.

Los CUH formados por Cx43 juegan un papel fundamental en el desarrollo de las células de la granulosa, según lo demuestra el análisis de los ovarios de ratones transgénicos carentes de $\mathrm{C} \times 43$ que mueren al momento de nacer probablemente como consecuencia de problemas cardiocirculatorios y ventilatorios (23). En los ovarios fetales provenientes de estos animales carentes de $\mathrm{Cx} 43$ y mantenidos en cultivo o implantados bajo la cápsula renal de hembras adultas, solamente ocurren las fases iniciales de la foliculogénesis, ya que, a diferencia de lo encontrado en los ratones silvestres, no hay folículos multilaminares (24-26). De manera similar, la maduración de los ovocitos de bovino es parcialmente impedida cuando se bloquea la expresión de la Cx43 mediante la transfección de los complejos cúmulo-ovocito con un adenovirus que expresa el ADN antisentido del ARNm de la Cx43 (27).
Los CUH comunican el gameto femenino en crecimiento con las células somáticas de su entorno en los folículos en desarrollo $(26,28)$. Esta comunicación intercelular heteróloga permite que las células del cúmulo mantengan al ovocito detenido durante la meiosis, ya que en algunas especies la maduración meiótica se reinicia coincidentemente con la interrupción de la comunicación intercelular (4). Al parecer hay una transferencia recíproca de señales intracelulares, a través de $\mathrm{CUH}$, entre las células somáticas y el ovocito, permitiendo que las células foliculares provean señales de crecimiento al ovocito y éste aporte señales reguladoras de la foliculogénesis (29-32). Los CUH entre las células de la granulosa son permeables a señales dependientes de AMPc (33) (Figura 2). Es posible que el AMPc sea la señal inhibitoria de la maduración que pasa a través de los $\mathrm{CUH}$ desde las células de la granulosa al ovocito (34). Por otra parte, en las células del cúmulo se generan ondas de $\mathrm{Ca} 2+$ que se transfieren al ovocito en respuesta a ATP (35), pero no parecen estar relacionadas con la maduración del ovocito. La Cx37 es un componente fundamental de estas interacciones heterocelulares, ya que se encuentra entre el ovocito en desarrollo y las células del cúmulo circundantes. Los ratones hembras carentes de Cx37 son infértiles y producen numerosos cuerpos lúteos alterados (12). El desarrollo folicular se detiene en la etapa preantral tipo 4 y el crecimiento del ovocito cesa en un diámetro que es sólo el $74 \%$ del tamaño folicular control, el ovocito se detiene en la etapa G2 del ciclo celular y no inicia la fase M (maduración meiótica), a menos que sea tratado con un inhibidor de fosfatasa (36). Actualmente se piensa que los CUH compuestos por Cx43 son regulados por mecanismos de compuerta (control de apertura o cierre del canal) activados por cambios en el estado de fosforilación de la Cx43 (6) y el grado de comunicación además depende del nivel de expresión de la Cx43 (37). 


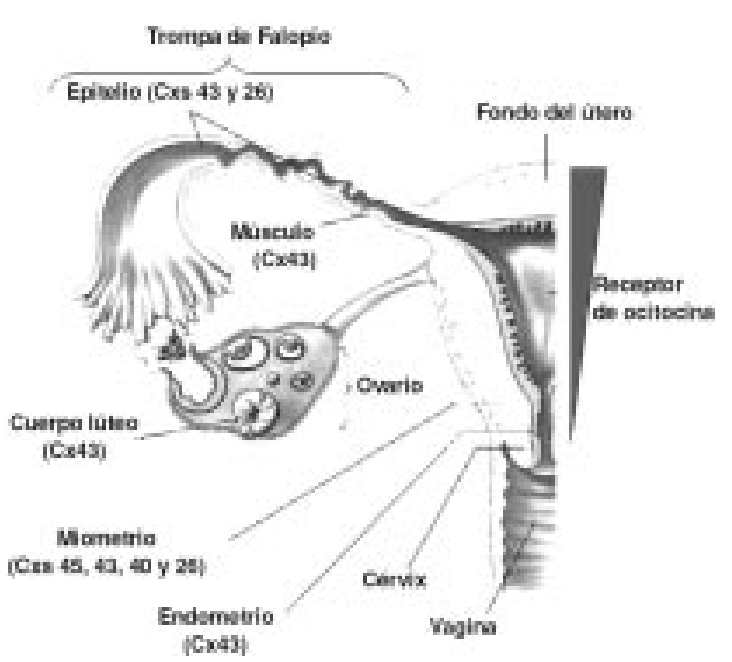

Figura 3. Diagrama de la distribución de conexinas (CXs) en distintos estratos celulares del tracto reproductor. El triángulo gris a la derecha del útero indica la densidad decreciente (desde el fondo del útero hacia el cérvix) de receptores de ocitocina en el miometrio.

\section{CUH Y CXS EN LAS TROMPAS DE FALOPIO}

Las células epiteliales de la trompa de Falopio humana, de rata, de ratón y de hámster, expresan las Cxs 43 y 26, mientras que las células de músculo liso expresan sólo Cx43 (38) (Figura 3). La cantidad de Cxs se correlaciona con la madurez sexual y los niveles de $\mathrm{Cx} 43$ se correlacionan con niveles altos de estrógenos (38). El papel de los $\mathrm{CUH}$ en este órgano no está claramente establecido, pero podría estar relacionado con la coordinación de la frecuencia de batido ciliar de las células epiteliales y/o con la coordinación de la contractilidad muscular. En ambos casos, la acción coordinada de estos tipos celulares presentes en la pared del oviducto favorecería el tránsito de gametos no fecundados para que ocurra la fertilización y el movimiento de los embriones hacia el útero para su implantación.

\section{CUH Y CXs EN EL ÚTERO}

En el útero, los CUH conectan a las células del músculo liso del miometrio y también a las células epiteliales del endometrio. En el caso del epitelio uterino se ha detectado comunicación intercelular asociada a la expresión de la Cx26 en la cámara implantacional de las conejas preñadas, pero no en las no preñadas o pseudo-preñadas (39), indicando la participación de señales del blastocisto en la inducción de la expresión de Cx26. Además, la expresión de las Cx26 y Cx43 en el endometrio es regulada de una manera diferencial por las hormonas ováricas durante el período pre y periimplantacional (40). En la mujer se han observado variaciones cíclicas en la expresión génica y los niveles de $\mathrm{C} \times 43$ en el endometrio asociadas al ciclo menstrual, y sugieren la posible participación de las Cxs como un marcador molecular de la ventana implantacional en la especie humana (41). Previo al inicio del trabajo de parto ocurre un gran aumento del número de CUH y en el grado de comunicación intercelular, que permitirían la contracción uterina coordinada (mayor fuerza por unidad de tiempo) durante este proceso (42). Esta característica del músculo uterino tiene particular relevancia debido a que las células musculares presentan una densidad decreciente de receptores de membrana para ocitocina en el miometrio desde el fondo del útero hacia el cérvix $(43,44)$ (Figura 3). Es decir, la activación de receptores de ocitocina en las células que expresan mayor densidad de receptores generaría señales intracelulares, que se propagarían a células con menos o carentes de receptores, y activarían la contracción de cada fibra muscular que invaden generando una respuesta contráctil coordinada hacia la vagina.

La identidad molecular responsable de la formación de los $\mathrm{CUH}$ en las células miometriales podría ser la $\mathrm{Cx} 43$ ya que se expresa en altos niveles (5) y su incremento podría relacionarse con el alza en los niveles de estriol que se detecta previo al parto (45). Sin embargo, se han identificado múltiples otras Cxs en estas células incluyendo a las Cxs 26,40 y 45 , cuyo papel aún no se esclarece (46-48). Se piensa que estas Cxs podrían formar canales mixtos (heteroméricos) con la Cx43 que co-localiza con, al menos, la Cx40 y la $\mathrm{C} \times 45$, en el músculo miometrial $(46,49,50)$. La expresión miometrial de las Cxs 43 y 26 es regulada de manera hormonal al inicio del parto. Al parecer el aumento en la relación de estrógenos versus progesterona es más importante que el aumento de cada hormona por separado.

Al menos en el caso de la Cx43, los mecanismos involucrados en el incremento de la proteína y de $\mathrm{CUH}$ detectados en el embarazo de término se asocian directamente al aumento de su ARNm como consecuencia de la regulación transcripcional y también a que se favorecería el tránsito de conexones de $\mathrm{C} \times 43$ hacia la membrana plasmática (51-53). La respuesta es órgano específica, ya que en dicho estado fisiológico, la cantidad de Cx43 en otros órganos prácticamente no se afecta (54). 
Los estudios realizados indican que las Cxs del miometrio, como del epitelio uterino, muestran diferentes patrones temporales y celulares de expresión durante importantes eventos del proceso reproductivo como la implantación, preñez, parto y post-parto $(5,48,55,56)$, sugiriendo que señales hormonales participan en la regulación de su expresión.

\section{PROYECCIONES}

Dada la reconocida importancia de los CUH en la respuesta de numerosos órganos, incluyendo los que forman parte del tracto reproductor femenino, parece necesario aclarar el posible papel de esta vía de comunicación intercelular en diversas patologías de dicho sistema, como infertilidad, embarazo tubárico y eclampsia. Además, el reenfoque de los diseños farmacológicos del tratamiento de numerosas patologías del aparato reproductor podrían considerar protocolos destinados a controlar el grado de comunicación intercelular entre células del tejido de interés.

\section{BIBLIOGRAFÍA}

1. Sáez JC, Berthoud VM, Brañes MC, Martínez AD, Beyer EC: Plasma membrane channels formed by connexins: their regulation and functions. Physiol Rev 2003; 83(4): 1359-60.

2. White TW, Paul DL: Genetic diseases and gene knockouts reveal diverse connexin functions. Annu Rev Physiol 1999; 61: 283-310.

3. Albertini DF, Anderson E: The appearance and structure of intercellular connections during the ontogeny of the rabbit ovarian follicle with particular reference to gap junctions. J Cell Biol 1974; 63(1): 234-50.

4. Gilula NB, Epstein ML, Beers WH: Cell-to-cell communication and ovulation. A study of the cumulus-oocyte complex. J Cell Biol 1978; 78(1): 5875.

5. Beyer EC, Kistler J, Paul DL, Goodenough DA: Antisera directed against connexin43 peptides react with a 43-kD protein localized to gap junctions in myocardium and other tissues. J Cell Biol 1989; 108(2): 595-605.

6. Granot I, Bechor E, Barash A, Dekel N: Connexin43 in rat oocytes: developmental modulation of its phosphorylation. Biol Reprod 2002; 66(3): 568-73.

7. Itahana K, Tanaka T, Morikazu Y, Komatu S, Ishida $\mathrm{N}$, Takeya T: Isolation and characterization of a novel connexin gene, Cx-60, in porcine ovarian follicles. Endocrinology 1998; 139(1): 320-29.

8. Manthey D, Bukauskas F, Lee CG, Kozak CA, Willecke K: Molecular cloning and functional ex- pression of the mouse gap junction gene connexin57 in human HeLa cells. J Biol Chem 1999; 274(21): 14716-23.

9. Mayerhofer A, Garfield RE: Immunocytochemical analysis of the expression of gap junction protein connexin 43 in the rat ovary. Mol Reprod Dev 1995; 41(3): 331-38.

10. Nuttinck F, Peynot N, Humblot P, Massip A, Dessy F, Flechon JE: Comparative immunohistochemical distribution of connexin 37 and connexin 43 throughout folliculogenesis in the bovine ovary. Mol Reprod Dev 2000; 57(1): 60-6.

11. Okuma A, Kuraoka A, lida $H$, Inai $T$, Wasano $K$, Shibata Y: Colocalization of connexin 43 and connexin 45 but absence of connexin 40 in granulosa cell gap junctions of rat ovary. J Reprod Fertil 1996; 107(2): 255-64.

12. Simon AM, Goodenough DA, Li E, Paul DL: Female infertility in mice lacking connexin 37 . Nature 1997; 385(6616): 525-29.

13. Valdimarsson G, De Sousa PA, Kidder GM: Coexpression of gap junction proteins in the cumulusoocyte complex. Mol Reprod Dev 1993; 36(1): 7-15.

14. Yoshizaki G, Patino R: Molecular cloning, tissue distribution, and hormonal control in the ovary of Cx41 mRNA, a novel Xenopus connexin gene transcript. Mol Reprod Dev 1995; 42(1): 7-18.

15. Gittens JE, Mhawi AA, Lidington D, Ouellette $Y$, Kidder GM: Functional analysis of gap junctions in ovarian granulosa cells: distinct role for connexin43 in early stages of folliculogenesis. Am J Physiol Cell Physiol. 2003; 284(4): 880-87.

16. Johnson ML, Redmer DA, Reynolds LP, GrazulBilska AT: Expression of gap junctional proteins connexin 43, 32, and 26 throughout follicular development and atresia in cows. Endocrine 1999; 10(1): 43-51.

17. Pérez-Armendariz EM, Sáez JC, Bravo-Moreno JF, Lopez-Olmos V, Enders GC, Villalpando I: Connexin43 is expressed in mouse fetal ovary. Anat Rec 2003; 271A(2): 360-67.

18. Granot I, Dekel N: Developmental expression and regulation of the gap junction protein and transcript in rat ovaries. Mol Reprod Dev 1997; 47(3): 231-9.

19. Wiesen JF, Midgley AR Jr: Changes in expression of connexin 43 gap junction messenger ribonucleic acid and protein during ovarian follicular growth. Endocrinology 1993; 133(2): 741-46.

20. Wiesen JF, Midgley AR Jr: Expression of connexin 43 gap junction messenger ribonucleic acid and protein during follicular atresia. Biol Reprod 1994; 50(2): 336-48.

21. Khan-Dawood FS, Yang J, Dawood MY: Expression of gap junction protein connexin-43 in the human and baboon (Papio anubis) corpus luteum. J Clin Endocrinol Metab 1996; 81(2): 835-42.

22. Grazul-Bilska AT, Redmer DA, Bilski JJ, JablonkaShariff A, Doraiswamy V, Reynolds LP: Gap junctional proteins, connexin 26,32 , and 43 in sheep 
ovaries throughout the estrous cycle. Endocrine 1998; 8(3): 269-79.

23. Reaume AG, de Sousa PA, Kulkarni S, Langille BL, Zhu D, Davies TC, Juneja C, Kidder GM, Rossant J: Cardiac malformation in neonatal mice lacking connexin43. Science. 1995; 267(5205): 1831-34.

24. Ackert CL, Gittens JE, O'Brien MJ, Eppig JJ, Kidder GM: Intercellular communication via connexin43 gap junctions is required for ovarian folliculogenesis in the mouse. Dev Biol 2001; 233(2): 58-70.

25. Juneja SC, Barr KJ, Enders GC, Kidder GM: Defects in the germ line and gonads of mice lacking connexin43. Biol Reprod 1999; 60(5): 1363-70.

26. Kidder GM, Mhawi AA: Gap junctions and ovarian folliculogenesis. Reproduction 2002; 123(5): 613-20.

27. Vozzi C, Formenton A, Chanson A, Senn A, Sahli R, Shaw P, Nicod P, Germond M, Haefliger JA: Involvement of connexin 43 in meiotic maturation of bovine oocytes. Reproduction 2001; 122(4): 619-28.

28. Anderson E, Albertini DF: Gap junctions between the oocyte and companion follicle cells in the mammalian ovary. J Cell Biol 1976; 71(2): 680-86.

29. Brower PT, Schultz RM. Intercellular communication between granulosa cells and mouse oocytes: existence and possible nutritional role during oocyte growth. Dev Biol 1982; 90(1): 144-53.

30. Eppig JJ: A comparison between oocyte growth in coculture with granulosa cells and oocytes with granulosa cell-oocyte junctional contact maintained in vitro. J Exp Zool 1979; 209(2): 345-53.

31. Vanderhyden BC, Caron PJ, Buccione R, Eppig JJ: Developmental pattern of the secretion of cumulusexpansion enabling factor by mouse oocytes and the role of oocytes in promoting granulosa cells differentiation. Dev Biol 1990; 140(2): 307-17.

32. Vanderhyden BC, Cohen JN, Morley P: Mouse oocytes regulate granulosa cell steroidogenesis. Endocrinology 1993; 133(1): 423-26.

33. Lawrence TS, Beers WH, Gilula NB: Transmission of hormonal stimulation by cell-to-cell communication. Nature 1978; 272(5623): 501-06.

34. Webb RJ, Marshall F, Swann K, Carroll J: Folliclestimulating hormone induces a gap junction-dependent dynamic change in [CAMP] and protein kinase A in mammalian oocytes. Dev Biol 2002; 246(2): 441-54.

35. Webb RJ, Bains H, Cruttwell C, Carroll J: Gapjunctional communication in mouse cumulus-oocyte complexes: implications for the mechanism of meiotic maturation. Reproduction 2002; 123(1): 41-52.

36. Carabatsos MJ, Sellitto C, Goodenough DA, Albertini DF: Oocyte-granulosa cell heterologous gap junctions are required for the coordination of nuclear and cytoplasmic meiotic competence. Dev Biol 2000; 226(2): 167-79.

37. Granot I, Dekel N: Phosphorylation and expression of connexin-43 ovarian gap junction protein are regulated by luteinizing hormone. J Biol Chem 1994; 269(48): 30502-09.
38. Hermoso M, Sáez JC, Villalón M: Identification of gap junctions in the oviduct and regulation of connexins during development and by sexual hormones. Eur J Cell Biol 1997; 74(1): 1-9.

39. Winterhager $E$, Brummer F, Dermietzel R, Hülser DF, Denker HW: Gap junction formation in rabbit uterine epithelium in response to embryo recognition. Dev Biol 1988; 126(1): 203-12.

40. Grummer R, Traub O, Winterhager E: Gap junction connexin genes cx26 and cx43 are differentially regulated by ovarian steroid hormones in rat endometrium. Endocrinology. 1999; 140(6): 2509-16.

41. Granot I, Dekel N, Bechor E, Segal I, Fieldust S, Barash A: Temporal analysis of connexin43 protein and gene expression throughout the menstrual cycle in human endometrium. Fertil Steril 2000; 73(2): 381-86.

42. Garfield RE, Sims S, Daniel EE: Gap junctions: their presence and necessity in myometrium during parturition. Science 1977; 198(4320): 958-60.

43. Fuchs AR, Fuchs F, Husslein P, Soloff MS: Oxytocin receptors in the human uterus during pregnancy and parturition. Am J Obstet Gynecol.; 1984: 150(6): 734-41.

44. Kitazawa T, Kajiwara T, Kiuchi A, Hatakeyama $H$, Taneike T: Muscle layer- and region-dependent distributions of oxytocin receptors in the porcine myometrium. Peptides 2001; 22(6): 963-74.

45. Di WL, Lachelin GC, McGarrigle HH, Thomas NS, Becker DL: Oestriol and oestradiol increase cell to cell communication and connexin 43 protein expression in human myometrium. Mol Hum Reprod. 2001; 7(7): 671-9.

46. Albrecht JL, Atal NS, Tadros PN, Orsino A, Lye SJ, Sadovsky Y, Beyer EC: Rat uterine myometrium contains the gap junction protein connexin 45, which has a differing temporal expression pattern from connexin43. Am J Obstet Gynecol 1996; 175(4Pt1): 853-58.

47. Orsino A, Taylor CV, Lye SJ: Connexin-26 and connexin43 are differentially expressed and regulated in the rat myometrium throughout late pregnancy and with the onset of labor. Endocrinology 1996; 137(5): 545-53.

48. Risek B, Gilula NB: Spatiotemporal expression of three gap junction gene products involved in fetomaternal communication during rat pregnancy. Development 1991; 123: 165-81.

49. Kilarski WM, Dupont E, Coppen S, Yeh HI, Vozzi C, Gourdie RG, Rezapour M, Ulmsten U, Roomans GM, Severs NJ: Identification of two further gapjunctional proteins, connexin40 and connexin45, in human myometrial smooth muscle cells at term. Eur J Cell Biol 1998; 75(1): 1-8.

50. Kilarski WM, RotheryYS, Roomans GM, Ulmsten U, Rezapour M, Stevenson S, Coppen SR, Dupont E, Severs NJ: Multiple connexins localized to individual gap-junctional plaques in human myometrial smooth muscle. Microsc Res Tech 2001; 54(2): 124-32. 
51. Grummer R, Chwalisz K, Mulholland J, Traub O, Winterhager E: Regulation of connexin26 and connexin43 expression in rat endometrium by ovarian steroid hormones. Biol Reprod 1994; 51(6): 1209-16.

52. Hendrix EM, Myatt L, Sellers S, Russell PT, Larsen WJ: Steroid hormone regulation of rat myometrial gap junction formation: effects on cx43 levels and trafficking. Biol Reprod 1995; 52(3): 547-60.

53. Risek B, Klier FG, Phillips A, Hahn DW, Gilula NB: Gap junction regulation in the uterus and ovaries of immature rats by estrogen and progesterone. J Cell Sci 1995; 108(Pt3): 1017-32.

54. Risek B, Guthrie S, Kumar N, Gilula NB: Modulation

Financiamiento: FONDECYT 3030066 (MCB), 1040804 (MJV) y 1030945 (JCS). of gap junction transcript and protein expression during pregnancy in rat. J Cell Biol 1990; 110(2): 269-82.

55. Pauken CM, Lo CW: Non overlapping expression of Cx43 and Cx26 in the mouse placenta and decidua: a pattern of gap junction gene expression differing from that in the rat. Mol Reprod Dev 1995; 41(2): 195-203.

56. Winterhager E, Grummer R, Jahn E, Willecke K, Traub O: Spatial and temporal expression of connexin26 and connexin43 in rat endometrium during trophoblast invasion. Dev Biol 1993; 157(2): 399-409. 